\title{
PRICING OF DELIVERY SERVICE IN A LOGISTICS NETWORK
}

\author{
Giuseppe Confessore, Daniele Corini, Giuseppe Stecca \\ Istituto di Tecnologie Industriali e Automazione - Consiglio Nazionale delle Ricerche, Area \\ della Ricerca di Roma Tor Vergata via Fosso del Cavaliere 100, 00133 Roma, ITALY \\ email: \{g.confessore,g.stecca\}@itia.cnr.it
}

\begin{abstract}
In this paper the problem of finding a computational fast method for pricing of the delivery service in a logistics network has been analyzed. The objective is to develop a supporting method for effective interaction among logistics service providers and customers.

We make use of an analytical function to predict the final total cost of distribution and to develop an interaction protocol that, using the function, leads to a final win-win solution in which the most of the customers have agreed to accept a delivery time comporting a reduction of total distribution costs. We also validated the method by use of a simulation software, evaluating the results in terms of cost-efficiency and stability.
\end{abstract}

\section{INTRODUCTION}

Logistic activities represent the connection point between goods production and fruition locations, which are separated by time and space (Pareschi et al, 2002). Global competition and tremendous technological progresses, in information technologies and logistics, impose to logistics operators a substantial reduction of their costs, particularly of those originated by the delivery process, when goods must be delivered to customers at right time in the right amounts and positions. The costs sustained by the distributor can be decomposed in three drivers: the variable cost of traveled distance, the variable cost of the vehicles, and their fixed cost. The class of problems studying the minimization of the transportation costs is commonly known as Vehicle Routing Problem (VRP); when customers ask for delivery in a specified time window, the sub-class to which it is referred to is the Vehicle Routing Problem with Time Windows constraints (VRPTW). The presence of a large amount of tight time windows leads to the increase of the number of needed vehicles, and a consequent increase of the transportation cost. On the contrary, if a customer would accept the delivery in a large time window, this would mean an increase on his costs, which originated either by the extra work for unload of the shipment in a unforeseen time window or by the loss of money of a missed sale due to unavailability of goods.

(So and Song, 1998) analyze the interrelations among pricing, delivery time guarantee and capacity expansion decisions. (Basu et al, 2004) extend spatial pricing theory for delivery service to encompass multizone plans. In (Campbell and Salvelsbergh, 2005) an incentive scheme specialized for home delivery service is 
proposed. In (Bianco et al, 2005) a formal decomposition of the vehicle routing problem and an interaction protocol is defined while in (Bernardi et al, 2004) a multiagent architecture is described. However, the high number of customers and the huge amount of possible solutions make difficult to resolve this problem to the optimum in a competitive interval of time, given that the problem belongs to the NP - hard in strong sense class of problems (Toth et al, 2002). In this paper we analyze the problem of finding a computational-fast method for the pricing of the delivery service in a logistics network, with the objective to develop a supporting method for effective interaction among logistics service providers and customers. The developed supporting method will help the implementation of an interaction protocol in which the logistics operator is able to price in a time-efficient manner the logistics service.

Given the opposite relationship between costs and windows widths, we have identified the trend of the costs as an analytical function, and then we have analyzed its ability to predict, with an adequately low error margin, the distribution costs using as the only input the measure of the time windows widths. Moreover, upon this analytical function we have built an algorithm for the solution of the delivery problem which leads to a final scenario where the most of the customers agree to broaden out their windows after an economical measurable saving on the transportation cost. On top of this algorithm, a multiagent system can be developed (Shen, and Norrie, 2001). Multiagent systems for supply chain integration and coordination (Durfee, Lesser, and Corkill, 1987; Gerber, Russ, and Klusch, 2003; Julka, Srinivasan, and Karimi, 2002; Karageorgos et al, 2003; Lee, and Whang 1999) can be thinked as a building block for collaborative networks (CamarinhaMatos and Afsarmanesh, 2004). The remainder of this paper is structured as follows. In Section 2 problem and model definition are introduced. In Section 3 the proposed solving approach is presented. In Section 4 conclusions and future works are discussed.

\section{PROBLEM AND MODEL DEFINITION}

The problem consists in finding a computational fast method for the pricing of the delivery service in a logistics network, with the objective to develop a supporting method for effective interaction among logistics service providers and customers. The developed supporting method will help the implementation of an interaction protocol in which the logistics operator is able to price in a time-efficient manner the logistics service.

We model the problem as follows:

Given a set of $n$ customers and an initial time window $W_{i}^{\infty}=\left[e^{0}, l_{i}^{0}\right]$ associated to each customer $i=1,2, \ldots, n$. Let $W_{i}=\left[e_{i}, l_{i}\right]$ be alternatives time windows associated to each customer $i=1,2, \ldots, n$ with $W_{i} \supseteq W_{i}^{0}$. Let $s_{i}^{0}=l_{i}^{0}-e_{i}^{0}$ and $s_{i}=$ $l_{i}-e_{i}$ be the width of the time windows in hours with $s_{i}^{0}, s_{i} \in\{1,2, \ldots, l\}$ where $l$ be the maximum time width. Let $\vec{w}^{0}=\left(W_{1}^{0}, W_{2}^{0}, \ldots, W_{n}^{0}\right)$, and $\vec{w}=\left(W_{1}, W_{2}, \ldots, W_{n}\right)$ vectors of the time windows. Let Let $n_{j}$ the number of customers having time 
windows width equal to $j$. Let moreover $S=\left(\sum_{j=1}^{l} n_{j} \cdot j\right) / n$. Given a market with a set of $n$ customers and a given resources, network structure, and good requests for customers, we can define $c_{i}\left(s_{i}\right) \geq 0$ as the cost for the customer $i$ for receiving delivery in a time window $W_{i}$ and $t c(\vec{w})$ the total cost of transportation for the logistics operator. We introduce the integer variables $e_{i}, l_{i} \in\{1,2, \ldots, l\}$. The problem is to find the combination of $\vec{w}=\left(W_{l}, W_{2}, \ldots, W_{n}\right)$ such that is

$$
\min t c(\vec{w})+\sum_{i=1}^{n} c_{i}\left(s_{i}\right)
$$

s.t.

$$
\begin{aligned}
& W_{i} \supseteq W_{i}^{0} \quad \forall i=1,2, \ldots, n \\
& W_{i}=\left[e_{i}, l_{i}\right] \quad \forall i=1,2, \ldots, n \\
& e_{i}<l_{i} \quad \forall i=1,2, \ldots, n \\
& e_{i}, l_{i} \in\{1,2, \ldots, l\}, \text { where } \vec{w}=\left(W_{1}, W_{2}, \ldots, W_{n}\right) \text { and } W_{i}^{0}=\left[e_{i}^{0}, l_{i}^{0}\right], \forall i=1,2, \ldots, n
\end{aligned}
$$

We notice that for each instance of $\vec{w}$ the solving of a VRPTW problem is needed.

\section{THE PROPOSED APPROACH}

To solve this problem we apply to the model the following procedure:

1. Find the analytical relationship between $t c$ and $S / l$;

2. Tune up of the function to prevent error margin;

3. Given an interaction protocol for the pricing of the delivery service, validate it;

4. Implement the interaction protocol in a multiagent based application.

\subsection{The Analytical Function}

The very first step of our research is made up by a set of tests, carried out with the goal of identifying an analytical function which is able to describe the distribution costs according to changes in customers' delivery time windows $W_{i}$.

We have considered 100 geographically-distributed customers (the position is defined by a couple of Cartesian reference points), with only 1 central depot, where the deliveries begin and end. Let $p$ the percentage of customers requesting for a tight time windows $p$, with $0 \leq p \leq 1$, and the measure of such tight time windows be $w$ hours, with $0 \leq w \leq l$; four scenarios have been considered:

Scenario 1: $p=0.25$;

Scenario 2: $p=0.50$;

Scenario $3: p=0.75$;

Scenario $4: p=1$. 
Customers not demanding for a tight time window are supposed to have $l$ hours wide windows, with $l=9$.

For each scenario, 4 sub-scenarios have been considered:

Sub Scenario a): $w=1$ hour;

Sub Scenario b): $w=3$ hours;

Sub Scenario c): $w=5$ hours;

Sub Scenario d): $w=7$ hours;

Each sub-scenario has been resolved using a VRPTW "ad hoc" solver based on Solomon I1 heuristic (Solomon et al, 1987): particularly, 10 runs of the solver have been performed for each sub-scenario, randomly assigning the tight delivery windows to different customers, but not changing windows start-times and duedates. A total of 160 runs have been resolved. The demand of each customer, based on Solomon " $R$ " instances, has been kept constant in all the runs, as well as the service time equal to 90 .

The outputs of the solver for each scenario are the total number of vehicles necessary to serve all the customers, and the total cost of distribution.

In Figure 1 a plot for each sub scenario $(w=1,3,5,7)$ is drawn, showing the total cost in function of the percentage $p$ of time window width. The total cost strongly increases with the increase of the percentage of customers, over the total, demanding for a tight delivery window: moreover, the differences in average total cost don't seem significant for requested delivery windows $w=\{3,5,7\}$ hours, while they are substantial between these 3 spans in which $w=1$ hour

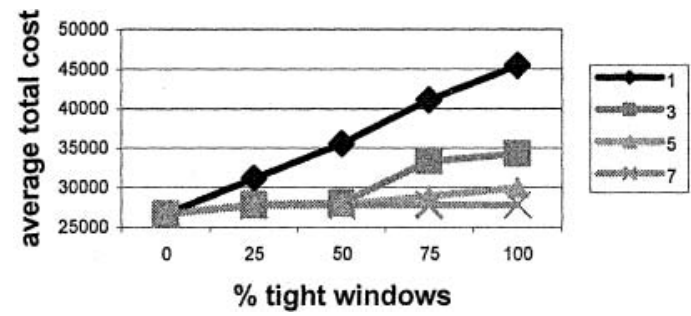

Figure 1 - Average total cost after percentages of tight windows

In order to find out the trend of the total cost in relationship with the $s / l$ ratio, we used the minimum squares method. Using this method we have chosen as regression function the logarithmical expression:

$$
\bar{t} c=a \ln (S / l)+b
$$

where $a$ and $b$ represent model constants with $a=-9395.7$ and $b=24382$.

In the moment of choosing which function, out of the four produced by the software for each scenario, we will be using for our future experiments, we decided to select the one, shown in Figure 2, produced by the fourth scenario, for the two following reasons: 
dependant on the suppliers regarding a component of high importance. But those assessments are no explicit part of the game - by purpose. By not asking a player these questions, he is forced to consider all important inputs to a decision to prevent experiencing negative results as long term effects.

\section{CONCLUSION}

The presented approach of PRIME uses two important elements to support the training process of business professionals: The trainees act in a network, which is very important to create a sufficient complexity in the VBE especially since human players act not as predictable as simulation models or artificial stakeholders, which are only included to fill the VBE and guarantee a sufficient amount of active players. Besides, the fact that human players have to deal with each other makes negotiations more realistic and even the personal aspect of inter-organizational collaboration is automatically present. The second major element is the free decision making, which omits querying the player to react on discrete events. Instead the players have to be pro-active and maintain an overview over their virtual environment and assess the current situation.

Based on this concept, and combined with a good concept of integrating games into corporate trainings, the PRIME consortium is developing a game, which has high potential to address not only the grave background of a learning tool, but also to mediate fun while playing. This way the PRIME game will increase the motivation of the trainees, since they will be able to play a game, even if it's just a supportive training activity.

\section{REFERENCES}

1. Baalsrud Hauge, J., Schwesig, M., Thoben, K.-D., Eschenbächer, J.: Business games - an effective tool for experiencing collaboration in production networks. pp. 30-37. In: Proceedings of the 9.th Workshop of IFIP W.G 5.7 - Special Interest Group on Experimental Interactive Learning in Industrial Management. Esbo, 2005.

2. Heide Smith, J.: The Problem of Other Players: In-game Cooperation as Collective Action. In: Proceedings of the DiGRA Digital Games Research Association Conference 2005

3. Herz, J.C., Macedonia, Michael R.: Computer Games and the Military: Two Views. Defense Horizons, Center for Technology and National Security Policy, National Defense University, April 2002

4. Linden Lab: Second Life. http://secondlife.com, Accessed: 09.03.2006

5. McLean, Charles, Jain, Sanjay, Lee, Y. T., Riddick, Frank, A: A Simulation and Gaming Architecture for Manufacturing Research, Testing, and Training. Proceedings in the IFIP 5.7 Advances in Production Management System Conference, Rockville, MD, September 18-21, 2005

6. Ondrejka, Cory: Changing Realities - User Creation, Communication, and Innovation in Digital Worlds. http://lindenlab.com/whitepapers/Changing_Realities_Ondrejka.pdf, 07.03.2006

7. Prensky, Marc: Digital Game-Based Learning. McGraw-Hill Companies, 2001

8. Schwesig, M., Thoben, K-D., Eschenbacher, J.: A Simulation Game Approach to Support Learning and Collaboration in Virtual Organisations. In: Proceedings of the 6th IFIP Working Conference on Virtual Enterprises, PRO-VE'05, Valencia, 2005

9. Serious Gaming Initiative, http://www.seriousgames.org/, Accessed: 05.03 .2006

10. Thoben et al:: Training through gaming - applying a simulation based business game to train people for collaboration in virtual enterprises. Online Educa Berlin 2005, 11. International Conference on Technology Supported Learning \& Training, 2005 
Now, putting this latest value inside the function equation, we finally obtain the predicted total cost $\bar{t} c$ for each group as:

$$
\bar{t} c=a \ln (\bar{r} / l)+b
$$

Making use of the percentages and the widths decided in the previous phase, we build VRPTW instances, yet without any change in customers' service time, demand and position, still equal to Solomon's II ones.

We build 10 instances for each group of percentages, again assigning delivery windows to customers in a random way, and then we resolve them using VRPTW solver, which produces the total cost of transportation $t c$ and the number of vehicles for each instance.

Each group $\vec{t} c$ and the 10 (exact) $t c$ 's are compared, and a correction rate that makes $\bar{t} c$ able to predict (merely, overtop) all $t c$ 's is evaluated

Looking at the results produced after the 4 groups of percentages, it is possible to observe that $70 \%$ of $t c$ 's are correctly predicted by the function. Thus, just carry a little correction to $\bar{t} c$ (the maximum needed is of $6 \%$, what is more already expected), making our function able to predict $100 \%$ of results.

\subsection{The interaction protocol}

The developed cost function can be used as a tool for measuring the transportation cost based on received orders, and for computing price discounts during negotiation with customers. A basic interaction protocol exploiting these potentialities is structured as follows:

INIZIALIZATION:

$-i=1, j=1 ; k=1 ; \bar{t} c^{\prime}=0, \bar{t} c^{\prime \prime}=0$;

-- The $n$ customers send orders to the Logistics Operator.

-- Computation of the number $n_{j}$ of customers having time window width $j$

-- Computation of transportation cost $t c$ ' through the function and first assignment to customers as $t c^{\prime}{ }_{i}=t c^{\prime} / n$.

STEP 1:

IF: $n_{j}-k>0$

$-n_{j}=n_{j}-k$

$-n_{j+1}=n_{j+1}+k$

-- Computation of new total cost of transportation, $t c$ ", and of the discount $\Delta=\bar{t} c^{\prime}-\bar{t} c^{\prime \prime}$

-- Send a propose to customers having time window width equal to $j$ a discount of $\Delta / n_{j}$

IF: at least $m>0$ customers exist for whom is valid $c_{i}(j)>c_{i}(j+1)-\Delta$

\section{ELSE:}

-- Enlarge time windows for such customers: $s_{i}=s_{i}+1$

\section{ELSE:}

$$
\text { -- Set } k=k+1 \text { and goto STEP } 1
$$




\section{STEP 2:}

$$
\text { IF: } \begin{aligned}
j & <l \\
& - \text { Computation of new } n_{j}+1 \text { and } n_{j}, \\
& -- \text { Set } j=j+1 \text {; goto STEP } 1
\end{aligned}
$$

\section{ELSE:}

$$
\begin{aligned}
& \text {-- Computation of new } n_{l-s} \text { and } n_{l} \\
& \text {-- Computation cost } t c \text { ' related to final numbers } n_{l}, n_{2}, \ldots, n_{l}
\end{aligned}
$$

\section{STOP}

\subsection{Simulation}

With the aim of validating the interaction protocol, we implemented it on a simulation software, building a model which reproduces its steps in order to reach a market result where the final percentages of customers asking for tight delivery windows are much less than initial ones, having the customers negotiated to broaden up their windows after a consistent saving. A total of 40 runs have been resolved. In Table 1 we show results of two runs. If the customers' cost structure is feasible, the negotiation will always lead to a final decrease of total costs. In the table this results can be argued from the medium time window width $S$.

Table1 - Examples of negotiation results

\begin{tabular}{|c|c|c|c|c|}
\hline \multirow{2}{*}{$j$} & \multicolumn{2}{|c|}{ Example 1 } & \multicolumn{2}{c|}{ Example 2 } \\
\cline { 2 - 5 } & Original $n_{j}$ & Final $n_{j}$ & Original $n_{j}$ & Final $n_{j}$ \\
\hline \hline 1 & 50 & 49 & 12 & 11 \\
\hline 2 & 0 & 0 & 11 & 8 \\
\hline 3 & 20 & 16 & 11 & 8 \\
\hline 4 & 0 & 0 & 11 & 8 \\
\hline 5 & 20 & 13 & 11 & 5 \\
\hline 6 & 0 & 0 & 11 & 2 \\
\hline 7 & 10 & 2 & 11 & 6 \\
\hline 8 & 0 & 9 & 11 & 4 \\
\hline 9 & 0 & 11 & 11 & 48 \\
\hline$S$ & 2.8 & 3.47 & 4.96 & 6.26 \\
\hline
\end{tabular}

Comparing the algorithm-made with the solver-made results, we can observe that our method has been able to reduce the number of customers asking for a tight, and troublesome, delivery window, carrying a real benefit to the whole system. Furthermore, the final total cost of distribution is always a little higher than the solver one, which means that the algorithm won't ever produce a lower cost than the real, needed one (produced by the solver), avoiding a loss to the distributor. 


\section{CONCLUSION}

In this paper we have found a computational-fast method for the pricing of the delivery service in a logistics network, with the objective to develop a supporting method for effective interaction among logistics service providers and customers. We have developed and tested an analytical function which is able to predict transportation costs and we have shown an interaction protocol for customers and logistics operator to decrease total distribution costs. Future works will regard the ability of the analytical function to predict changes in quantity requests, the use of a better solving algorithm to find function parameters and the development of a multiagent system implementing the described interaction protocol.

\section{REFERENCES}

1. Basu A; Ingene CA, Mazumdar T. "The pricing of delivery services". Journal Of Regional Science, vol. 44 , no. 4, 743-772, 2004.

2. Bianco L, Confessore L, Stecca G. "A multi-agent Model for distribution problems in logistics systems", in Proceedings of 18th International Conference on Production Research ICPR18, Fisciano(Sa) Italy, July 31 th - August 4 th 2005.

3. Bernardi D, Confessore G, Stecca G. "A multiagent model integrating inventory and routing processes", in Virtual Enterprises and Collaborative Networks, L.M. Camarinha-Matos (eds.). Kluwer Academic publishers 2004; 107-114.

4. Camarinha-Matos L M, Afsarmanesh $\mathrm{H}$. "The emerging discipline of collaborative networks". In Virtual Enterprises and Collaborative Networks, L.M. Camarinha-Matos (eds.): Kluwer Academic publishers 2004.

5. Campbell AM, Savelsbergh M. "Incentive Schemes for Attended Home Delivery Services". Accepted to Transportation Science, publication forthcoming, 2005.

6. Durfee, EH, Lesser VR, Corkill, DD. "Coherent cooperation among communicating problem solvers". In IEEE Trans. Computers, C-36, 1275, 1987.

7. Gerber A, Russ C, Klusch M. "supply web co-ordination by an agent-based trading network with integrated logistics services". In Electronic Commerce Research and Applications 2, Elsevier Science Ltd., 2003.

8. Julka N, Srinivasan R, Karimi I. Agent Based Supply Chain Management 1 \& 2, Computers And Chemical Engineering 26, Elsevier Science Ltd., 2002.

9. Karageorgos A, Mehandjiev N, Weichhart G, Hammerle A. "Agent-Based Optimisation Of Logistic And Production Planning", in Engineering Applications of Artificial Intelligence 16, Elsevier Science Ltd., 2003.

10. Lee H, Whang S. Decentralized. "Multi-Echelon Supply Chains: Incentives and Information". in Management Science Vol. 45, No.5, May 1999.

11. Pareschi A, Persona A, Ferrari, E, Regattieri, A. Logistica integrata e flessibile. Editrice Esculapio, 2002.

12. Shen W, Norrie DH, Barthès J-PA. Multi-Agent systems for concurrent intelligent design and manufacturing. London and New York: Taylor \& Francis, 2001.

13. So KC, Song IS. "Price, delivery time guarantees and capacity selection". In European Journal of Operational Research 111, 28-49, 1998.

14. Solomon MM. "Algorithms for the Vehicle Routing and Scheduling Problems with Time Windows constraints". in Operations Research 35, 254-265, 1987.

15. Toth P, Vigo D. The Vehicle Routing Problem. Siam, 2002. 